\title{
NUMBER OF ZEROS OF A POLYNOMIAL IN A GIVEN DOMAIN
}

\author{
A. EBADIAN, M. BIDKHAM AND M. ESHAGHI GORDJI
}

\begin{abstract}
In this paper, we obtain results concerning the bound for the number of zeros for the polynomial $p(z)$ which generalize earlier well-known result due to Bidkham and Dewan [On the zeros of a polynomial, Numerical Methods and Approximation Theory III, Niš (1987), 121-128] and Mohammad [On the zeros of polynomials, Amer. Math. Monthly, 72 (1965), 631-633]. We also obtain result for location of zeros of polynomial $p(z)=\sum_{i=0}^{m} \frac{a_{i}}{(i !)^{\lambda}} z^{i}+a_{n} z^{n}, a_{n} \neq 0,0 \leq m \leq n-1, \lambda \geq 0$.
\end{abstract}

\section{Introduction and Statement of Results}

The problems in the analytic theory of polynomials concerning the number of zeros in a circle have been frequently investigated.

Over many decades, a large number of research papers, e.g., [2, 4, 5, 6, 7, 8, 9, 10, 11, 12, $14,16,17,19,20]$ have been published.

For the number of zeros in a circle we have the following result due to Singh [18].

Theorem A. Let $p(z)=\sum_{i=0}^{n} a_{i} z^{i}$ be a polynomial of degree $n$ such that $\min _{0 \leq i \leq n}\left|a_{i}\right| \geq 1$ and $\max _{0 \leq i \leq n-1}\left|a_{i}\right| \leq$ $\left|a_{n}\right|$, then

$$
n\left(\frac{R}{k}\right) \leq \frac{2 \log \left\{(n+1)\left|a_{n}\right| R^{n}\right\}}{\log k} \quad(k>1)
$$

where $n(r)$ is the number of zeros of $p(z)$ in $|z| \leq r$ and

$$
R=\max \left\{\left|\frac{a_{n-1}}{a_{n}}\right|,\left|\frac{a_{n-2}}{a_{n}}\right|^{\frac{1}{2}},\left|\frac{a_{n-3}}{a_{n}}\right|^{\frac{1}{3}}, \ldots\right\} .
$$

The above theorem was later improved by Rahman [15].

For the class of polynomial with real coefficients we have the following theorem due to Mohammad [13].

Corresponding author: A. Ebadian.

2010 Mathematics Subject Classification. 26C10, 12D10, 30D15.

Key words and phrases. Polynomials, zeros of polynomials. 
Theorem B. Let $p(z)=\sum_{i=0}^{n} a_{i} z^{i}$ be a polynomial of degree $n$ such that $a_{n} \geq a_{n-1} \geq \cdots \geq a_{1} \geq$ $a_{0}>0$, then the number of zeros of $p(z)$ in $|z| \leq \frac{1}{2}$ does not exceed

$$
1+\frac{1}{\log 2} \log \frac{a_{n}}{a_{0}}
$$

Bidkham and Dewan [3] generalized the above theorem for different classes of polynomials and proved the following.

Theorem C. Let $p(z)=\sum_{i=0}^{n} a_{i} z^{i}$ be a polynomial of degree $n$ such that $a_{n} \leq a_{n-1} \leq \cdots \leq a_{k+1} \leq$ $a_{k} \geq a_{k-1} \geq \cdots \geq a_{0}$ for some $k, 0 \leq k \leq n$, then the number of zeros of $p(z)$ in $|z| \leq \frac{1}{2}$ does not exceed

$$
\frac{1}{\log 2}\left\{\log \frac{\left|a_{n}\right|+\left|a_{0}\right|-a_{n}-a_{0}+2 a_{k}}{\left|a_{0}\right|}\right\} \text {. }
$$

Theorem D. Let $p(z)=\sum_{i=0}^{n} a_{i} z^{i}$ be a polynomial of degree $n$ with complex coefficients. If for some real $\beta,\left|\arg a_{i}-\beta\right| \leq \alpha \leq \frac{\pi}{2}, 0 \leq i \leq n$ and for some $0<t \leq 1$,

$$
\left|a_{0}\right| \leq t\left|a_{1}\right| \leq \cdots \leq t^{k}\left|a_{k}\right| \geq t^{k+1}\left|a_{k+1}\right| \geq \cdots \geq t^{n}\left|a_{n}\right|
$$

$0 \leq k \leq n$, then the number of zeros of $p(z)$ in $|z| \leq \frac{1}{2}$ does not exceed

$$
\frac{1}{\log 2} \log \left\{\frac{2 t^{k+1}\left|a_{k}\right| \cos \alpha+2 t \sin \alpha \sum_{i=0}^{n} t^{i}\left|a_{i}\right|-t^{n+1}\left|a_{n}\right|(\cos \alpha+\sin \alpha-1)}{t\left|a_{0}\right|}\right\}
$$

In this paper, we generalize Theorems B,C and D for polynomials with real and complex coefficients. More precisely, we prove.

Theorem 1. Let $p(z)=\sum_{i=0}^{n} a_{i} z^{i}$ be a polynomial of degree $n$ such that $a_{n} \leq a_{n-1} \leq \cdots \leq a_{k+1} \leq$ $a_{k} \geq a_{k-1} \geq \cdots \geq a_{0}$ for some $k, 0 \leq k \leq n$, then the number of zeros of $p(z)$ in $|z| \leq \frac{R}{2}(R>0)$ does not exceed

$$
\frac{1}{\log 2}\left\{\log \frac{\left|a_{n}\right| R^{n+1}+\left|a_{0}\right|+R^{k}\left(a_{k}-a_{0}\right)+R^{n}\left(a_{k}-a_{n}\right)}{\left|a_{0}\right|}\right\} \text { for } R \geq 1
$$

and

$$
\frac{1}{\log 2}\left\{\log \frac{\left|a_{n}\right| R^{n+1}+\left|a_{0}\right|+R\left(a_{k}-a_{0}\right)+R^{n}\left(a_{k}-a_{n}\right)}{\left|a_{0}\right|}\right\} \text { for } R \leq 1
$$


For $R=1$, Theorem 1 reduces to inequality (1.3). For $R=1, k=n$ and $a_{0}>0$ it reduces to inequality (1.2).

Theorem 2. Let $p(z)=\sum_{i=0}^{n} a_{i} z^{i}$ be a polynomial of degree $n$ with complex coefficients. If for some real $\beta,\left|\arg a_{i}-\beta\right| \leq \alpha \leq \frac{\pi}{2}, 0 \leq i \leq n$, and for some $R>0$,

$$
\left|a_{0}\right| \leq R\left|a_{1}\right| \leq \cdots \leq R^{k}\left|a_{k}\right| \geq \cdots \geq R^{n}\left|a_{n}\right|
$$

$0 \leq k \leq n$, then the number of zeros of $p(z)$ in $|z| \leq \frac{R}{2}(R>0)$ does not exceed

$$
\frac{1}{\log 2}\left\{\log \frac{2 R^{k+1}\left|a_{k}\right| \cos \alpha+2 R \sin \alpha \sum_{i=0}^{n} R^{i}\left|a_{i}\right|-R^{n+1}\left|a_{n}\right|(\cos \alpha+\sin \alpha-1)}{R\left|a_{0}\right|}\right\}
$$

For $R=1, k=n$ and $\alpha=\beta=0$, the above theorem reduces to inequality (1.2).

Regarding the bound for zeros of the polynomial $p(z)=\sum_{i=0}^{m} \frac{a_{i}}{(i !)^{\lambda}} z^{i}+a_{n} z^{n}, a_{n} \neq 0,0 \leq m \leq$ $n-1, \lambda \geq 0$, we have able to prove the following.

Theorem 3. Let $p(z)=\sum_{i=0}^{m} \frac{a_{i}}{(i !)^{\lambda}} z^{i}+\frac{a_{n}}{(n !)^{\lambda}} z^{n}, 0 \leq m \leq n-1, \lambda \geq 0$ be a polynomial of degree $n$ with complex coefficients such that $\left|a_{i}\right| \leq(i !)^{\lambda}\left|a_{n}\right|, 0 \leq i \leq m$, then all zeros of $p(z)$ lie in $|z|<r$, where $r$ is unique positive root of equation

$$
|z|^{n-m}-|z|^{n-m-1}=(n !)^{\lambda}
$$

\section{Lemmas}

The following lemma is well-known Jensen's theorem (see page 208 of [1]).

Lemma 2.1. Assume that $f$ is analytic in a disk $|z| \leq R$, but not identically zero. Assume also $f(0) \neq 0$. Let $f$ have zeros $\left\{a_{k}\right\}, k=1,2, \ldots, n$ in $|z| \leq R$, then

$$
\frac{1}{2 \pi} \int_{0}^{2 \pi} \log \left|f\left(R e^{i \theta}\right)\right| d \theta-\log |f(0)|=\sum_{i=1}^{n} \log \frac{R}{\left|a_{i}\right|} .
$$

One get easily from Lemma 2.1, the following.

Lemma 2.2. If $f(z)$ is regular, $f(0) \neq 0$ and $|f(z)| \leq M(r)$ in $|z| \leq r$, then the number of zeros of $f(z)$ in $|z| \leq \frac{r}{2}$ does not exceed

$$
\frac{1}{\log 2}\left(\log \frac{M(r)}{|f(0)|}\right)
$$


Lemma 2.3. Let $p(z)=\sum_{i=0}^{n} a_{i} z^{i}$ be a polynomial of degree $n$ such that $\left|\arg _{i}-\beta\right| \leq \alpha \leq \frac{\pi}{2}$ for $i=0,1, \ldots, n$ for some real $\beta$, then for some $t>0$

$$
\left|t a_{i}-a_{i-1}\right| \leq|t| a_{i}|-| a_{i-1}|| \cos \alpha+\left(t\left|a_{i}\right|+\left|a_{i-1}\right|\right) \sin \alpha .
$$

The proof is omitted as it follows from the inequality (6) in [4].

\section{Proof of the Theorems}

Proof of Theorem 1. Consider the polynomial

$$
\begin{aligned}
g(z) & =(1-z) p(z) \\
& =-a_{n} z^{n+1}+a_{0}+\sum_{i=1}^{n}\left(a_{i}-a_{i-1}\right) z^{i} .
\end{aligned}
$$

For $|z| \leq R$, we have

$$
|g(z)| \leq\left|a_{n}\right| R^{n+1}+\left|a_{0}\right|+\sum_{i=1}^{k}\left(a_{i}-a_{i-1}\right) R^{i}+\sum_{i=k+1}^{n}\left(a_{i-1}-a_{i}\right) R^{i}
$$

which gives

$$
|g(z)| \leq\left|a_{n}\right| R^{n+1}+\left|a_{0}\right|+R^{k}\left(a_{k}-a_{0}\right)+R^{n}\left(a_{k}-a_{n}\right) \text { for } \quad R \geq 1
$$

and

$$
|g(z)| \leq\left|a_{n}\right| R^{n+1}+\left|a_{0}\right|+R\left(a_{k}-a_{0}\right)+R^{k}\left(a_{k}-a_{n}\right) \quad \text { for } \quad R \leq 1
$$

which further imply

$$
\left|\frac{g(z)}{g(0)}\right| \leq \frac{\left|a_{n}\right| R^{n+1}+\left|a_{0}\right|+R^{k}\left(a_{k}-a_{0}\right)+R^{n}\left(a_{k}-a_{n}\right)}{\left|a_{0}\right|} \quad \text { for } R \geq 1
$$

and

$$
\left|\frac{g(z)}{g(0)}\right| \leq \frac{\left|a_{n}\right| R^{n+1}+\left|a_{0}\right|+R\left(a_{k}-a_{0}\right)+R^{k}\left(a_{k}-a_{n}\right)}{\left|a_{0}\right|} \text { for } R \leq 1 .
$$

Applying Lemma 2.2 to $g(z)$, we get the number of zeros of $g(z)$ in $|z| \leq \frac{R}{2}$ does not exceed

$$
\frac{1}{\log 2}\left\{\log \frac{\left|a_{n}\right| R^{n+1}+\left|a_{0}\right|+R^{k}\left(a_{k}-a_{0}\right)+R^{n}\left(a_{k}-a_{n}\right)}{\left|a_{0}\right|}\right\} \text { for } R \geq 1
$$

and

$$
\frac{1}{\log 2}\left\{\log \frac{\left|a_{n}\right| R^{n+1}+\left|a_{0}\right|+R\left(a_{k}-a_{0}\right)+R^{k}\left(a_{k}-a_{n}\right)}{\left|a_{0}\right|}\right\} \text { for } R \leq 1 .
$$


As the number of zeros of $p(z)$ in $|z| \leq \frac{R}{2}$ does not exceed the number of zeros of $g(z)$ in $|z| \leq \frac{R}{2}$, the theorem follows.

Proof of Theorem 2. Consider

$$
F(z)=(R-z) p(z)=-a_{n} z^{n+1}+R a_{0}+\sum_{i=1}^{n}\left(R a_{i}-a_{i-1}\right) z^{i} .
$$

For $|z| \leq R$, we have

$$
|F(z)| \leq\left|a_{n}\right| R^{n+1}+R\left|a_{0}\right|+\sum_{i=1}^{n}\left|R a_{i}-a_{i-1}\right| R^{i} .
$$

Using Lemma 2.3, for $|z| \leq R$, we get

$$
\begin{aligned}
|F(z)| \leq & \left|a_{n}\right| R^{n+1}+R\left|a_{0}\right|+\sum_{i=1}^{n}|R| a_{i}|-| a_{i-1}|| R^{i} \cos \alpha+\sum_{i=1}^{n}\left(R\left|a_{i}\right|+\left|a_{i-1}\right|\right) R^{i} \sin \alpha \\
= & \left|a_{n}\right| R^{n+1}+R\left|a_{0}\right|+\sum_{i=1}^{k}\left(R\left|a_{i}\right|-\left|a_{i-1}\right|\right) R^{i} \cos \alpha+\sum_{i=k+1}^{n}\left(\left|a_{i-1}\right|-R\left|a_{i}\right|\right) R^{i} \cos \alpha \\
& +\sum_{i=1}^{n}\left(R\left|a_{i}\right|+\left|a_{i-1}\right|\right) R^{i} \sin \alpha \\
= & 2 R^{k+1}\left|a_{k}\right| \cos \alpha+2 R \sin \alpha \sum_{i=0}^{n} R^{i}\left|a_{i}\right|-R\left|a_{0}\right|(\cos \alpha+\sin \alpha-1)-R^{n+1}\left|a_{n}\right|(\cos \alpha+\sin \alpha-1) \\
\leq & 2 R^{k+1}\left|a_{k}\right| \cos \alpha+2 R \sin \alpha \sum_{i=0}^{n} R^{i}\left|a_{i}\right|-R^{n+1}\left|a_{n}\right|(\cos \alpha+\sin \alpha-1) .
\end{aligned}
$$

Further, proceeding on the same lines of the proof of Theorem 1, the proof of Theorem 2 can be completed.

Proof of Theorem 3. For $|z| \leq 1$, the conclusion of Theorem 3 is evident. If $|z|>1$, then

$$
\begin{aligned}
|p(z)| & =\left|\frac{a_{n}}{(n !)^{\lambda}} z^{n}+\frac{a_{m}}{(m !)^{\lambda}} z^{m}+\cdots+a_{1} z+a_{0}\right| \\
& \geq \frac{\left|a_{n}\right||z|^{n}}{(n !)^{\lambda}}-\left\{\frac{\left|a_{m}\right|}{(m !)^{\lambda}}|z|^{m}+\cdots+\left|a_{1}\right||z|+\left|a_{0}\right|\right\} \\
& =\left|a_{n}\right|\left|z^{n}\right|\left\{\frac{1}{(n !)^{\lambda}}-\left[\frac{1}{(m !)^{\lambda}} \frac{\left|a_{m}\right|}{\left|a_{n}\right|} \frac{1}{|z|^{n-m}}+\cdots+\left|\frac{a_{1}}{a_{n}}\right| \frac{1}{|z|^{n-1}}+\frac{\left|a_{0}\right|}{\left|a_{n}\right|} \frac{1}{|z|^{n}}\right]\right\}
\end{aligned}
$$

Now if $\left|a_{i}\right| \leq(i !)^{\lambda}\left|a_{n}\right|, i=0, \ldots, m$ is assumed, we conclude

$$
\begin{aligned}
|p(z)| & \geq\left|a_{n} \| z\right|^{n}\left\{\frac{1}{(n !)^{\lambda}}-\left[\frac{1}{|z|^{n-m}}+\cdots+\frac{1}{|z|^{n}}\right]\right\} \\
& >\left|a_{n} \| z\right|^{n}\left\{\frac{1}{(n !)^{\lambda}}-\sum_{i=0}^{\infty} \frac{1}{|z|^{i}}+\left(1+\frac{1}{|z|}+\cdots+\frac{1}{|z|^{n-m-1}}\right)\right\} \\
& =\left|a_{n} \| z\right|^{n}\left\{\frac{1}{(n !)^{\lambda}}-\frac{|z|}{|z|-1}+\frac{|z|}{|z|-1}-\frac{\frac{1}{|z|^{n-m}}}{1-\frac{1}{|z|}}\right\}
\end{aligned}
$$




$$
\begin{aligned}
& =\left|a_{n}\right||z|^{n}\left\{\frac{1}{(n !)^{\lambda}}-\frac{1}{(|z|-1)|z|^{n-m-1}}\right\} \\
& =\frac{\left|a_{n}\right||z|^{n}}{(n !)^{\lambda}|z|^{n-m-1}(|z|-1)}\left\{(|z|-1)|z|^{n-m-1}-(n !)^{\lambda}\right\} \\
& =\frac{\left|a_{n}\right||z|^{n}}{(n !)^{\lambda}|z|^{n-m-1}(|z|-1)} H(|z|) \\
& \geq 0 \text { for }|z| \geq r .
\end{aligned}
$$

Hereby proving Theorem 3.

\section{References}

[1] Lars V. Ahlfors, Complex Analysis, 3rd ed., McGraw-Hill.

[2] H. Alzer, Bounds for the zeros of polynomials, Complex Variables, Theory Appl., 8(2001), 143-150.

[3] M. Bidkham and K. K. Dewan, On the zeros of a polynomial, Numerical Methods and Approximation Theory III, Niš (1987), 121-128.

[4] M. Dehmer, On the location of zeros of complex polynomials, Jipam, 4(2006), 1-13.

[5] M. Dehmer, Schranken fr die Nullstellen komplexwertiger Polynome, Die Wurzel, 8 (2000), 182-185.

[6] N. K. Govil and Q. I. Rahman, On the Eneström-Kakeya Theoryem, Tôhoku Math. J., 20(1968), 126-136.

[7] F. Heigl, Neuere Entwicklungen in der Geometrie der Polynome, Jber. Deutsch. Math.-Verein., 65 (1962/63), 97-142.

[8] P. Henrich, Applied and computational complex analysis, Volume I, Wiley Classics Library Edition, (1988).

[9] F. Kittaneh, Bounds for the zeros of polynomials from matrix inequalities, Arch. Math., 81(2003), 601-608.

[10] S. Lipka, ber die Abgrenzung der Wurzeln von algebraischen Gleichungen, Acta. Litt. Sci. (Szeged), 3 (1927), 73-80.

[11] M. Marden, Geometry of polynomials, Mathematical Surveys. Amer. Math. Soc., Rhode Island, 3 (1966).

[12] Q. G. Mohammad, Location of the zeros of polynomials, Amer. Math. Monthly, 74 (1967), 290-292.

[13] Q. G. Mohammad, On the zeros of polynomials, Amer. Math. Monthly, 72 (1965), 631-633.

[14] N. Obreschkoff, Verteilung und Berechnung der Nullstellen reeller Polynome, Hochschulbcher fr Mathematik. 55. Berlin, VEB Deutscher Verlag der Wissenschaften, 1963.

[15] Q. I. Rahman, On the zeros of a class of polynomials, Proc. Nat. Inst. Sci. India, 22(1956), 137-139.

[16] Q. I. Rahman and G. Schmeisser, Analytic Theory of Polynomials. Critical Points, Zeros and Extremal Properties, Clarendon Press, 2002.

[17] D. M. Simeunovic', On the location of the zeros of polynomials, Math. Morav., 2 (1998), 91-96.

[18] S. K. Singh, On the zeros of a class of polynomials, Proc. Nat. Inst. Sci. India, 19(1953), 601-603.

[19] W. Specht, Die Lage der Nullstellen eines Polynoms, Mathematische Nachrichten, 15 (1956), 353-374.

[20] E. C. Westerfield, A new bound for the zeros of polynomials, Amer. Math. Monthly, 40 (1933), 18-23. J. Inequal. Pure and Appl. Math., 7(1) Art. 26, 2006.

Department of Mathematics, Faculty of Sciences, Urmia University, Urmia, Iran.

E-mail: a.ebadian@urmia.ac.ir

Department of Mathematics, Faculty of Natural Sciences, Semnan University, Semnan, Iran.

E-mail: mbidkham@semnan.ac.ir

Department of Mathematics, Faculty of Natural Sciences, Semnan University, Semnan, Iran.

E-mail: majid.eshaghi@gmail.com 